\title{
TOPOLOGICAL FOUNDATION AND KINETICS OF TEXTURE CONTROLLED GRAIN GROWTH
}

\author{
G. Abbruzzese*, I. Heckelmann*, K. Lücke* \\ Centro Sviluppo Materiali, Roma, Italy \\ * Institut für Metallkunde und Metallphysik, RWTH Aachen, \\ Kopernikusstr. 14, D-5100 Aachen, FRG
}

\begin{abstract}
In the present paper first a statistical theory of 2-dimensional grain growth for the textureless case based on first principles - the von Neumann Mullins equation and the topological grain size - grain sides relationship - is described. Then it is shown that the latter relationship follows from two fundamental topological principles, the principles of complete and random surface covering, which are shown to be responsible also for other empirical topological 2-D and 3-D relationships (e.g. Weaire equation). Finally, textures are introduced into the topological discussion.
\end{abstract}

\section{INTRODUCTION}

In order to completely describe grain growth one has to know the true evolution of a function $\mathrm{p}\left(\mathrm{M}_{1}, \mathrm{M}_{2}, \ldots \mathrm{M}_{4}\right)$ which gives the distribution of grain shape and size identified by the parameters $M_{1}, M_{2}, \ldots M_{k}$. In the rather common case of equiaxed grains it is usual to make an assumption on the shape of the grains which allows, with a good approximation, to treat the grains by only a single parameter namely the grain size, here expressed by the grain radius $\mathbf{R}$. In this case only the time evolution of a size distribution $\varphi(R, t)$ must be considered. In the most general case of texture presence this requires to know the time evolution of the different grain size distributions corresponding to the various orientation classes.

There are two types of assumptions one needs for obtaining a quantitative description of grain growth kinetics:

(a) Physical assumptions as those for the expressions of the driving force and the rate of grain boundary motion. They were applied to an individual grain in the textureless case by von Neumann using first principles ${ }^{1,2}$. It turned out that here the number $\mathbf{n}$ of grain sides plays an important role in the kinetics.

(b) Topological assumptions which are required to transform the n-dependence of the grain growth kinetics into an R-dependence. These are as important as the physical assumptions but treated less thoroughly in literature. This point will here be emphasized.

\section{2-DIMENSIONAL CASE WITHOUT TEXTURE}

The physical expressions for driving force and grain boundary 
velocity are:

$$
p=\frac{\gamma}{\lambda}=\gamma \frac{d \theta}{d s} ; \quad v=m p=\frac{m \gamma}{\lambda}
$$

where $\gamma=$ specific grain boundary energy; $m=$ grain boundary mobility; $\lambda=$ radius of curvature; $\boldsymbol{\theta}=$ inclination of the grain boundary element. It is further assumed that these boundaries only meet at triple points where they form $120^{\circ}$ angles. This represents the equilibrium condition which in grain growth should be fulfilled quite well.

For a polygonal grain one can obtain a simple expression for the growth rate of the grain area by calculating the mean curvature with the total change $\Delta \theta$ of the inclination $\theta$ occurring by going along all boundaries around this grain. $\Delta \theta$ is obtained by substracting from the total change $2 \pi$ an angle of $60^{\circ}$ for each corner (which is due to the $120^{\circ}$ intersections, see also Fig.1):

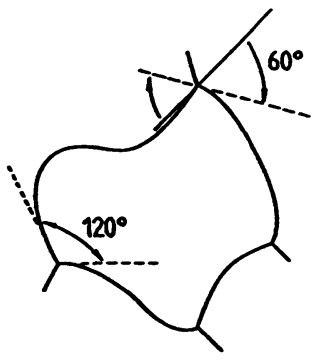

Fig. 1: Example of an individual grain

$$
\frac{d A}{d t}=-m \gamma\left(2 \pi-n \frac{\pi}{3}\right)=\frac{\pi}{3} m \gamma(n-6)
$$

or by replacing the grain area $A$ by $A=\pi R^{2}$

$$
\frac{d R}{d t}=\frac{m \gamma}{6 R}(n-6)
$$

This "von Neumann - Mullins equation" contains two parameters describing a grain, namely $n$ and $R$, and in order to treat the time evolution of the bidimensional distribution $\varphi(n, R)$, one has to have an independent equation for the rate of changing of the number of corners $\mathrm{dn} / \mathrm{dt}$ based on first principles. However, at present such equation is not available which is also due to the discontinuous character of this parameter.

Therefore, in order to solve the problem of grain growth without further assumptions one has to look for a correlation between $\mathbf{n}$ and $\mathbf{R}$. However, since one of these parameters changes discontinuously and the other continuously, this relationship cannot have unique but only statistical character. Dividing the grains in size classes $i$ one can substitute for each class $i$ the average value of the scattered $n$ values (see Fig. 2) thus obtaining a continuous function $\bar{n}_{i}=\bar{n}_{i}\left(R_{i}\right)$. It can be inserted in the von Neumann - Mullins equation (Eq.(3)) averaged over all 


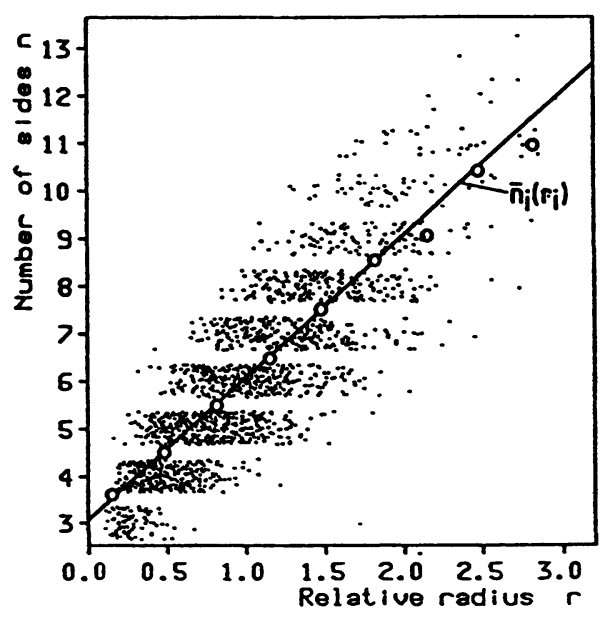

Fig.2: Normalized radius and number of sides of $\sim 2000$ grains of an Al 3\% Mg specimen (points); average number of sides $\bar{n}$, for each radius class $i$ vs. $r_{i}$ (circles). The straight line is obtained by linear regression.

grains of the size class $\mathbf{i}$ but of various side numbers $\mathbf{n}$ yielding

$$
\frac{d R_{t}}{d t}=\frac{m \gamma}{6 R_{i}}\left(n_{t}\left(R_{t}\right)-6\right)
$$

valid for each size class $i$.

In order to apply Eq.(4) the right expression of $\bar{n}_{i}\left(R_{i}\right)$ must be known. In the literature often the existence of a linear relationship like

$$
\bar{n}_{i}=a_{0}+a r_{i} \quad r_{i}=R_{i} / \bar{R}
$$

is mentioned. Since in a 2-D network like a grain microstructure where only three lines meet at a vertex the average number of corners per grain over the whole microstructure must be six, one has

$$
\bar{n}=\sum_{i} \varphi_{i} \bar{n}_{i}=6=a_{0}+a
$$

with $\varphi_{i}$ as the normalized frequency of grains belonging to the size class $i$. In the literature very many results were presented which do not fulfill this condition or use the wrong type of averages, e.g. the most probable numbers of sides. Therefore, here Eq.(5) has been checked by thorough own experimental investigations for very large numbers of grains (see Fig.2). Application of the Least Square Method yielded a linear equation given very accurately by

$$
\bar{n}_{i}=3+3 r_{i}
$$

One can see that it fulfills Eq.(6) and that for the smallest grains $\left(r_{i}=0\right) \bar{n}_{i}=3$ is obtained reflecting the fact that the disappearing grains are 3 -sided ones.

Introducing Eq.(7) in the averaged von Neumann - Mullins equation (4) 
leads to the equation

$$
\frac{d R_{i}}{d t}=\frac{1}{2} m \gamma\left(\frac{1}{\bar{R}}-\frac{1}{R_{i}}\right)
$$

given by Hillert ${ }^{3}$ for 2-dimensional grain growth, but derived on a more heuristic basis. Now it shows more clearly the physical meaning of its origin. This equation together with a continuity equation can be used to describe the time evolution of the grain size distribution $\varphi(R, t)$ in the textureless case ${ }^{4}$ :

$$
\frac{\partial \varphi(R, t)}{\partial t}=-\frac{\partial}{\partial R}\left[\varphi(R, t) \frac{d R}{d t}\right]
$$

Eqs.(8) and (9) give a complete solution of the problem of quantitative description of 2-D grain growth. It is derived from first principles except for the assumption of the empirical linear relationship Eq.(7). In the next section, however, it will be shown that also this relationship is based on fundamental topological principles.

\section{UNDERLYING TOPOLOGICAL PRINCIPLES}

The agreement of the linear relationship with the average values in Fig. 2 shows that this is not only an empirical law but expresses some topological facts. Therefore an (approximate) derivation of Eq.(4) will be given on the basis of fundamental topological principles. A model will be used in which polygonal grains are substituted by circles of equivalent area. Two basic assumptions are made:
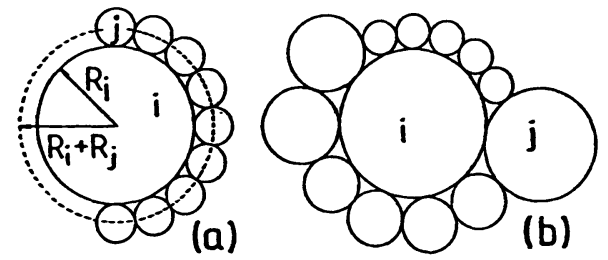

Fig. 3: (a) Grain of size $R_{1}$ surrounded by grains of one size $R_{\text {. }}$. (b) Grain of size $R_{i}$ surrounded by grains of different sizes $R_{f}$.

(i) Total surface covering (Fig.3a). The grains $j$ with radius $R_{j}$ which can surround grain $i$ with radius $R_{i}$ are calculated by

$$
n_{i j}=\frac{2 \pi\left(R_{i}+R_{j}\right)}{2 R_{j}} \simeq 3+3 \frac{R_{i}}{R_{j}}
$$

$\pi$ is valid for grains being strictly circular whereas for polygonal grains the value 3 is more correct.

(ii) Random surface covering (Fig.3b). The average number of neighbours of a grain $i$ is given by

$$
\bar{n}_{i}=\sum_{j} w_{f} n_{t j}=\sum_{j} \frac{\varphi_{f} R_{j}}{\bar{R}} \frac{3\left(R_{i}+R_{j}\right)}{R_{j}}=3+3 \frac{R_{i}}{\bar{R}}=3+3 r_{i} .
$$


Here represents

$$
w_{j}=\varphi_{f} 2 \pi R_{j} / \sum_{j} \varphi_{j} 2 \pi R_{j}=\varphi_{f} R_{f} / \bar{R}
$$

the fraction of the total grain surface occupied by grains of the size $R_{\mathrm{j}}$. This means Eq.(11) is valid for the case that the surface of any grain $i$ is occupied by grains $j$ in a random way, i.e. only according to the surface fraction of the $j$ grains.

The above derivation reveals the two first principles which determine the validity of Eq.(7), namely the principles of total and random surface covering being approximately expressed by Eqs.(10) and (12). On the other hand if one wants to describe a microstructure where topological correlations are present, i.e. that the principle of random surface covering is not fulfilled, the linear relationship is maybe no longer valids.

These topological principles can also be applied to the 3-D case in order to derive a relationship between size $R_{i}$ and number of faces $\overline{\mathbf{n}}_{i}^{\mathrm{F}}$ of the grains. Considering a sphere $i$ covered by spheres $j$ one obtains

$$
\begin{gathered}
n_{i j}^{F}=\frac{4 \pi\left(R_{t}+R_{j}\right)^{2}}{\pi R_{i}^{2}} ; \quad w_{j}^{F}=\frac{\varphi_{j} 4 \pi R_{j}^{2}}{\sum_{j} \varphi_{j} 4 \pi R_{f}^{2}} \\
\bar{n}_{t}^{F}=\sum_{j} w_{j}^{F} n_{t j}^{F}=4+8 \frac{R_{t}}{R_{c}}+4 \frac{R_{i}^{2}}{R R}
\end{gathered}
$$

where one has

$$
R_{c}=\bar{R}^{2} / \bar{R}=\bar{R}+\sigma^{2} / \bar{R}
$$

$\left(\sigma^{2}=\right.$ distribution variance). One sees that the relationship Eq.(14) is no longer linear but quadratic in $R$ and depends on the shape of the size distribution $\left(\sigma^{2}\right)$. Fig. 4 shows the so far most complete 3-D data, measured by Rhines and Patterson $^{6}$ on Aluminium. Here it has to be pointed out that all the data for the 3-D case in the literature, also those from Rhines and Patterson are always discussed in terms of a linear relationship. However, even the rather rough straight line shown in Fig.4 represents a non-linear law in the $\left(\bar{n}^{F}, R\right)$ space, namely $\overline{\mathbf{n}}^{\mathrm{F}} \approx \mathbf{R}^{1,33}$. Moreover, the downward deviation of the experimental data for low $\overline{\mathrm{n}}^{\mathrm{F}}$ values shows that the exponent of the real law should be higher than 1.33 . In Fig.5 the data from Rhines and Patterson are transformed to linearly divided axes. Here it can be seen easily that the relation is rather quadratic than linear. This is in agreement with the above predictions of a quadratic law (Eq.(14)) with $\overline{\mathbf{n}}^{\mathrm{F}}(\mathrm{R}=0)=4$ (tetrahedron).

From the principles of total and random surface covering also the aver- 
average boundary length $\bar{L}_{i}$ of the grains as a function of the grain size $i$
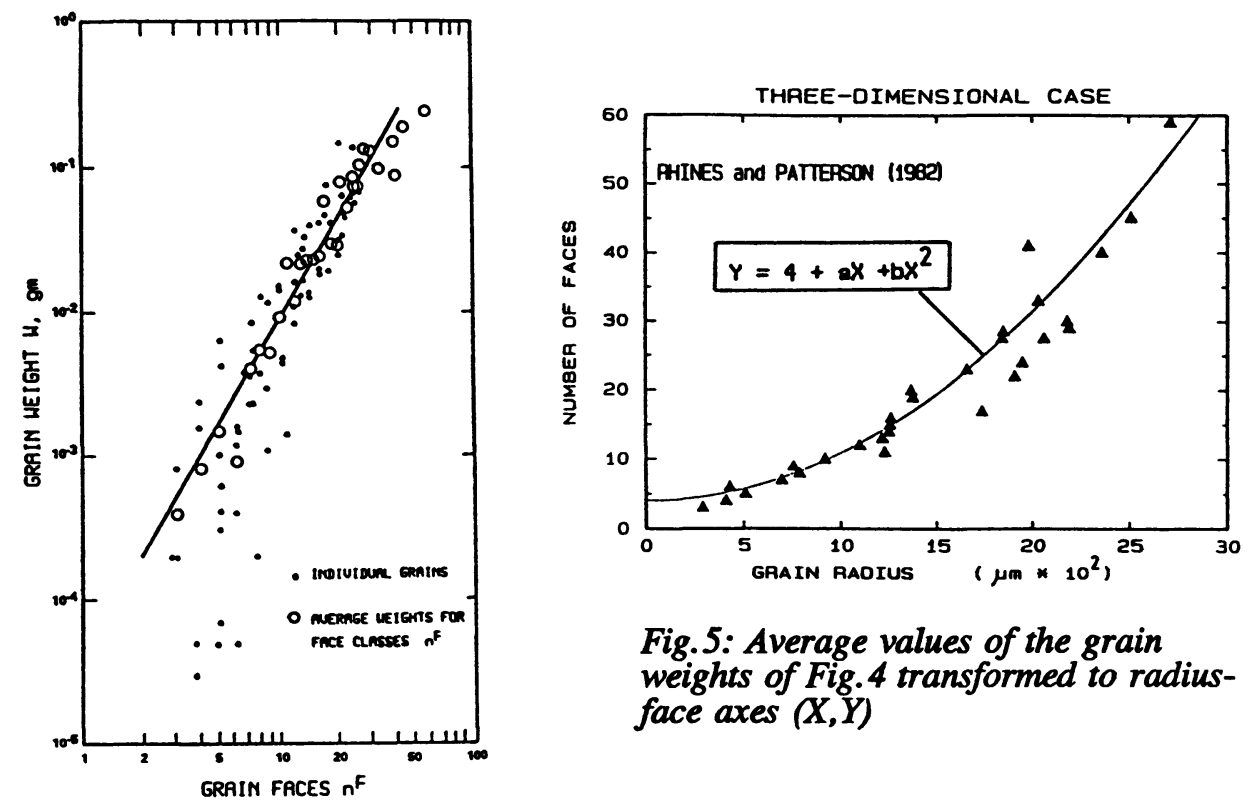

Fig.5: Average values of the grain weights of Fig. 4 transformed to radiusface axes $(X, Y)$

Fig.4: Grain weight vs. number of faces per grain (measurements on Aluminium from Rhines and Patterson, 1982)

can be calculated. If a grain $i$ is surrounded only by grains of the size $j$, the length of its boundary is given with Eq.(10) by

$$
L_{i j} \simeq \frac{2 \pi R_{i}}{n_{i j}} \simeq \frac{6 R_{i}}{n_{i j}}=2 \frac{R_{i} R_{j}}{R_{i}+R_{j}}
$$

and by applying the random surface covering postulate it follows for the average length of grains $\mathrm{i}$ :

$$
\begin{aligned}
& \bar{\ell}_{i}=\frac{\bar{L}_{i}}{\bar{R}}=\frac{1}{\bar{R}} \frac{\sum_{j} w_{j} n_{v} L_{i}}{\sum_{j} w_{j} n_{v}}=2 \frac{r_{i}}{1+r_{i}} \\
& \bar{L}_{i}\left(R_{i}=0\right)=0 ; \quad \bar{L}_{i}\left(R_{i} \rightarrow \infty\right) \rightarrow 2 \bar{R}
\end{aligned}
$$

Such type of relationship (Eq.(17)) was also observed experimentallys. 
Finally it should be mentioned that the above topological principles are also determining other topological relationships, e.g. the correlation between number $n$ of sides of a grain and the average number $\bar{n}_{\mathbf{a}}$ of sides of the first neigbhours in a 2-D microstructure:

$$
\bar{n}_{s}=5+\frac{6+\sigma_{n}^{2}}{n}
$$

$\left(\sigma_{n}^{2}=\right.$ variance of the distribution of grain corners). This relationship has empirically been found by Aboav and Weaire, but no clear derivation based on fundamental assumptions. is reported yet in the literature. As will be shown in another paper, also this type of rather complex correlation can be derived from the above fundamental topological principles. All these agreements demonstrate that the grain structure even in rather large detail is determined by the principles of complete and random surface coverages.

\section{INTRODUCTION OF TEXTURE FOR THE 2-D CASE}

In the case that different orientations are present the linear relationship and the fundamental law $\overline{\mathbf{n}}=6$ for the whole microstructure are still valid. Even if one applies the above topological principles Eqs.(10) and (12) to a single orientation class $(\mathrm{H})$ one still gets a linear relationship

$$
\bar{n}_{t}^{H}=3+3 \frac{R_{i}^{H}}{\bar{R}},
$$

since these principles concern only the grain geometry independent on the orientation. But one sees that the average number of sides of the given orientation can be different from 6 as much as $\mathbf{R}^{\mathrm{H}}$ deviates from $\mathbf{R}$ :

$$
\bar{n}^{H}=3+3 \frac{\bar{R}^{H}}{\bar{R}} \not \equiv 6
$$

Integrated over all orientation classes, one obtains again the fundamental law $\overline{\mathbf{n}}=6$ for the whole microstructure.

$$
\bar{n}=\sum_{H} \varphi^{H} n^{H}=\sum_{H} \varphi^{H}\left[3+3 \frac{\bar{R}^{H}}{\overline{\bar{R}}}\right]=6,
$$

with $\varphi^{\mathrm{H}}$ being the relative frequency of grains belonging to the orientation class $\mathrm{H}$. The contribution of different texture classes to the total linear relationship is schematically shown in Fig.6. Here the simple case of only two orientation classes with different sizes was chosen so that their radii do not superimpose. Such microstructures can be found e.g. during secondary recrystallization where one has primary and secondary grains of rather different sizes. 


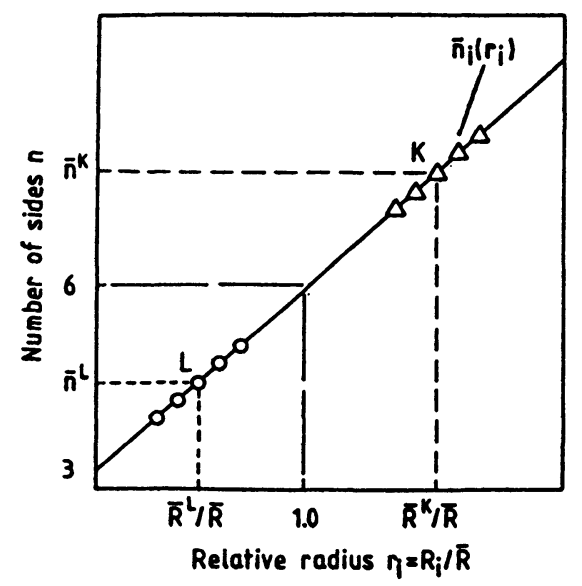

Fig. 6: Contribution of two orientation classes $L$ and $K$ with different mean sizes $\boldsymbol{R}^{L}$ and $\boldsymbol{R}^{\boldsymbol{R}}$ to the total linear relationship. The average number of sides of the grains belonging to different size and orientation classes are presented by symbols

Furthermore it follows for all other topological rules which are derived from these principles, e.g. the grain size dependence of the grain boundary length or the Weaire-Equation (Eqs.(17),(19)) that they are not only valid for the whole microstructure, but also for grains of a single orientation class.

\section{CONCLUSIONS}

Any statistical theory of grain growth must contain some topological assumptions capable to take into account all the complex features of grain arrangement. Here it has been shown that important topological laws, as e.g. the relationship between grain size and number of sides (faces in 3-D), the dependence of the mean grain boundary length on the grain size and the Weaire-equation, can be derived from fundamental topological principles, namely the postulates of total and random surface covering. It is further shown that these relationships are also valid if, instead of the whole microstructure, only grains belonging to individual orientation classes are considered.

\section{REFERENCES}

1. Von Neumann, discussion to C.S. Smith, Metal Interfaces, Am. Soc. for Metals, 65, Cleveland (1952)

2. W.W. Mullins, J. Appl. Phys. 27, 900 (1956)

3. M. Hillert, Acta metall. 13, 227 (1965)

4. G. Abbruzzese, K. Lücke, Acta metall. 34, 905 (1986)

5. G. Abbruzzese, I. Heckelmann, K. Lücke, Acta metall. mater. in press

6. F.N. Rhines, B.R. Patterson, Metall. Trans. 13A, 985 (1982)

7. D. Weaire, Metallography, 7, 157 (1974) 